\title{
Acoustic-driven Oscillations of a Bubble in a Narrow Gap
}

\author{
Jacqueline Mifsud $^{1}$, Duncan A. Lockerby ${ }^{1}$, Yongmann M. Chung ${ }^{1}$, Gordon Jones ${ }^{2}$ \\ ${ }^{1}$ School of Engineering \\ University of Warwick, Coventry CV4 7AL, United Kingdom \\ j.mifsud@warwick.ac.uk; d.lockerby@warwick.ac.uk \\ ${ }^{2}$ Waters Corporation \\ Stamford Avenue, Wilmslow SK9 4AX, United Kingdom \\ gordon_jones@waters.com
}

\begin{abstract}
The complex interaction of an acoustic cavitation bubble with a boundary is widely exploited in practical applications. In ultrasonic cleaning, acoustic cavitation is used to eliminate surface contamination and particulate debris following manufacturing. However, very little is known about the fundamental physics behind such processes. Furthermore, bubble collapse in the presence of two or more boundaries is considerably different than the traditional problem of collapse near a single rigid wall. In this study, we combine the complex pressure-driven dynamics of acoustic cavitation in confinement. We focus on the dynamics and jet development of an oscillating gas bubble $\left(R_{0}=50 \mu \mathrm{m}\right)$ driven by a strong low-frequency acoustic field ( $p_{a c}=95 \mathrm{kPa}$ and $\left.f_{a c}=20 \mathrm{kHz}\right)$. Since inertial oscillations are responsible for most of the practical effects of acoustic cavitation, we select an acoustic driving frequency lower than the natural frequency of the bubble to demonstrate the transient response. We present results from compressible volume-of-fluid simulations with consideration of viscosity and surface tension conducted in the open-source framework of OpenFOAM. We find that the presence of a secondary opposing wall intensifies the jet velocities at collapse. We provide a detailed description of the flow inside the gap, particularly the complex interaction between flow deflected by the confining boundaries and the oscillatory driving pressure. By varying the gap height and the position of bubble inception, we observe significantly different bubble collapse dynamics across the considered parameter space, which can be categorised in three distinct jetting regimes according to relevant nondimensional parameters. We also demonstrate how an increased liquid viscosity alters the collapse mechanism and jet formation through a representative case. The results presented in this study have immediate consequences in acoustic cavitation applications involving confinement, since through careful choice of driving conditions and fluid properties, the desired behaviour to suit the application can be achieved.
\end{abstract}

Keywords: bubble dynamics; acoustic cavitation; confinement; narrow gap; OpenFOAM; volume-of-fluid

\section{Introduction}

Ever since the discovery of cavitation over a hundred years ago by Lord Rayleigh, cavitation microbubbles have been harnessed in an arsenal of applications [1] including: i) medical applications such as non-invasive therapy and drug delivery [2,3] ii) microfluidic (lab-on-chip) devices [4]; and iii) ultrasonic cleaning of surfaces [5]. Although most practical engineering applications of cavitation involve bubble clouds, their complex interactions cannot be accurately modelled without prior knowledge and understanding of the dynamics of individual bubbles.

In a uniform flow, a cavitation microbubble will remain spherical and its behaviour is well described by classical analytical models such as the Rayleigh-Plesset Equation (RPE) [6]. However, in practical applications, the pulsation of a cavitation bubble is often constrained by nearby boundaries exhibiting more complex dynamics, which are not entirely understood. Bubbles near boundaries collapse asymmetrically forming jets, the speed and direction of which are highly influenced by i) the type of boundary (e.g. a rigid/elastic boundary, free surface or an adjacent bubble) and ii) the proximity of the boundary [7]. Therefore, realistic configurations cannot be described by empirical equations such as the RPE but require more sophisticated numerical models.

Bubble oscillations in narrow gaps have received attention only recently [8-10], but none of these studies considered acoustic cavitation dynamics confined by rigid boundaries. To the authors' best knowledge, this study is the first to address this knowledge-gap. Computational Fluid Dynamics (CFD) modelling is used to inform the development of the surrounding flow-field and splitting and jet formation events for various bubble positions inside the gap. Previous finite-volume studies have shown that the accurate prediction of the bubble radius after multiple collapse-expansion cycles is difficult to achieve [11-14]; either because the bubble is too small at the collapse and/or because the highly distorted interface 
disintegrates quickly after collapse. In this study, our focus is therefore the bubble shape dynamics until first collapse. The driving pressure is modelled by an oscillatory boundary condition.

\section{Bubble Model and Governing Equations}

The compressible Navier-Stokes (NS) equations are solved using an algebraic Volume-of-Fluid (VOF) method within the open-source CFD framework of OpenFOAM to predict the flow conditions both inside and outside the bubble [11]. The model is based on the native solver compressibleInterFoam, a pressure-based solver for compressible two-phase flow that captures the interface evolution between the two phases. VOF is an interface-capturing method known for the ease with which it handles complex splitting and jetting phenomena, topologies synonymous with multiphase flows $[15,16]$.

\subsection{Governing equations}

The equations of motion are formulated for a single fluid having a common velocity field $\boldsymbol{U}$, pressure field $p$ and density field $\rho$. An advection equation for the volume fraction $\alpha$ is solved to capture the gas-liquid interface (Eq.(1)). The governing system of equations can be written as follows [11]:

$$
\begin{gathered}
\frac{\partial \alpha_{l}}{\partial t}+\nabla \cdot\left(\alpha_{l} \boldsymbol{U}\right)+\nabla \cdot\left(\boldsymbol{U}_{r} \alpha_{l} \alpha_{g}\right)=\alpha_{l} \alpha_{g}\left(\frac{\psi_{g}}{\rho_{g}}-\frac{\psi_{l}}{\rho_{l}}\right)\left(\frac{\mathrm{D} p}{\mathrm{D} t}\right)+\alpha_{l} \nabla \cdot \boldsymbol{U}, \\
\frac{\partial \rho}{\partial t}+\nabla \cdot(\rho \boldsymbol{U})=0, \\
\frac{\partial(\rho \boldsymbol{U})}{\partial t}+\nabla \cdot(\rho \boldsymbol{U} \otimes \boldsymbol{U})=-\nabla p+\nabla \cdot \mathbb{T}+f_{\sigma} .
\end{gathered}
$$

The volume fraction $\alpha_{l}$ acts as a phase indicator as follows:

$$
\alpha_{l}=\left\{\begin{array}{cl}
0 & \text { for gas } \\
0<\alpha_{l}<1 & \text { for interface } \\
1 & \text { for liquid. }
\end{array}\right.
$$

In Eq. (1), $\boldsymbol{U}_{\boldsymbol{r}}$ is the relative velocity at the interface and acts as an artificial compressibility term to ensure a sharp interface between the two phases. The parameters $\psi_{g}$ and $\psi_{l}$ are the nonlinear compressibilities of the gas and liquid respectively, computed as $\psi=D \rho / D p$ depending on the chosen equation of state. In Eq.(3), $\mathbb{T}$ is the viscous stress tensor and $f_{\sigma}$ is the surface tension force, modelled according to the Continuum Surface Force (CSF) method of Brackbill [17].

\subsection{Barotropic closure}

The above system of governing equations Eqs.(1-3) is closed by the barotropic equation of state for each phase. The nonlinear compressibility of both the gas and liquid phases is considered. For the liquid, the density $\rho_{l}$ is related to the pressure $p$ through the Tait equation of state:

$$
\rho_{l}=\rho_{0, l}\left(\frac{p+B}{p_{0}+B}\right)^{1 / \gamma_{l}},
$$

where $\rho_{0, l}$ and $p_{0}$ are the density and pressure of the liquid at atmospheric conditions, respectively; the Tait exponent $\gamma_{l}=$ 7 and the Tait pressure $B=331 \mathrm{MPa}$ as in [18].

Similarly, the pressure $p$ and density of the gas $\rho_{g}$ are related through the polytropic equation of state: 


$$
\rho_{g}=\rho_{0, g}\left(\frac{p}{p_{0}}\right)^{1 / \gamma_{g}}
$$

where $\rho_{0, g}$ and $p$ are the density and pressure of the gas (air) inside the bubble at atmospheric conditions and $\gamma_{g}=1.4$ is the specific heat ratio for air. The use of the barotropic equations of state implies that the pressure is independent of temperature, therefore the energy equation becomes redundant.

The fluid type is identified based on the value of the volume fraction $\alpha$. The liquid is taken as the primary phase such that $\alpha_{l}=1$ represents water whilst $\alpha_{l}=1-\alpha_{g}=0$ corresponds to the gaseous phase. The physical properties, namely the density $\rho$ and dynamic viscosity $\mu$, of the respective phases can then be averaged as follows:

$$
\begin{aligned}
& \rho=\alpha_{l} \rho_{l}+\left(1-\alpha_{l}\right) \rho_{g}, \\
& \mu=\alpha_{l} \mu_{l}+\left(1-\alpha_{l}\right) \mu_{g} .
\end{aligned}
$$

The fluid properties are those for water as a liquid, and air as a gas at atmospheric conditions $\left(\sim 20^{\circ} \mathrm{C}\right)$.

\subsection{Simulation setup}

The schematic diagram in Fig. 1a depicts the simulation setup and the boundary conditions. To alleviate some of the computational cost of the problem the domain is axisymmetric. The inclusion of viscosity in the model requires that both walls be supplemented with no-slip boundary conditions for velocity. A zero-gradient boundary condition is implemented for the volume fraction $\alpha$ and pressure $p$ at the walls. An oscillatory pressure field $p_{\infty}=p_{0}-p_{a c} \sin \left(2 \pi f_{a c} t\right)$ is imposed at the gap outlet, characterised by the acoustic pressure amplitude $p_{a c}$ and the driving frequency $f_{a c}$. The use of barotropic equations of state Eqs. (5) and (6) means that the pressure $p$ must return a non-zero positive value to ensure that the density is also non-zero. For this reason, the acoustic pressure amplitude $p_{a c}$ is limited to fractions of the ambient pressure: $p_{a c}=$ $k p_{0}$ where $k<1$. This prevents the pressure becoming negative in the rarefactional half-cycle of the signal [19]. At the gap outlet, the volume fraction $\alpha$ and velocity $\boldsymbol{U}$ are assigned a zero-gradient boundary condition.

Fig. 1b shows the relevant geometrical parameters. Both the gap height $H_{c}$ and the distance $h$ between the initial position of the bubble centre and the primary wall are varied. The parameters $L_{c}$ and $H_{c}$ together define the aspect ratio of the gap $L_{c} / H_{c}$. The domain of length $L_{c}=1 \mathrm{~mm}$ in the radial direction and a variable height $H_{c}$ is discretised using recursive refinement on an initial uniform grid with resolution of 100 cells in the radial direction. The initial coarse grid is refined recursively five times to obtain a minimum resolution of $\Delta x \approx 0.3 \mu \mathrm{m}$ in the bubble region. This high resolution is required to sufficiently resolve the bubble at its minimum volume.

a)

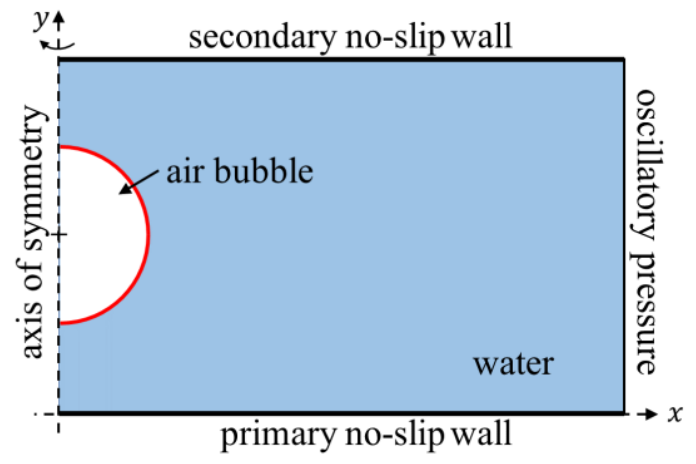

b)

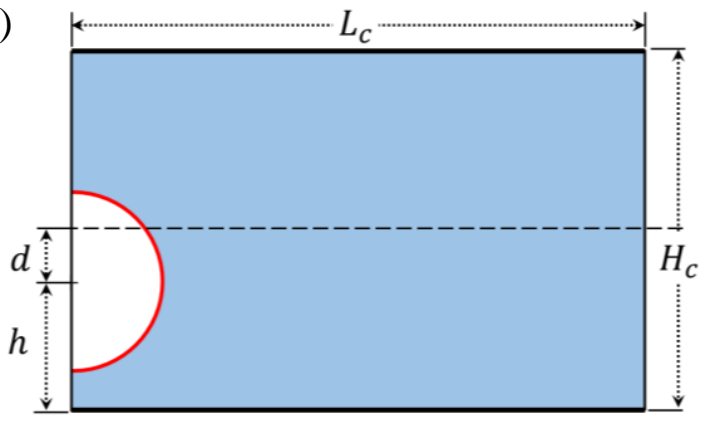

Fig. 1: a) Simulation setup and boundary conditions; b) Relevant geometrical parameters. 
Existing studies on the influence of confinement on bubble dynamics assume an undriven, initially small, bubble at high pressure to match laser-induced or spark-generated cavitation bubbles in experiments [8-10,20]. The initial conditions in this study are the static equilibrium conditions. The equilibrium radius $R_{0}$ is the size at which the surface tension $\sigma$ at the gas-liquid interface is balanced by the pressure difference at the bubble wall:

$$
p_{B, 0}-p_{0}=\frac{2 \sigma}{R_{0}}
$$

where $p_{B, 0}$ is the initial pressure inside the bubble and $p_{0}$ is the atmospheric pressure. The simulations therefore start with an overpressure in the bubble prior to excitation by the externally applied pressure field $p_{\infty}$.

\subsection{Validation of the numerical model}

We use the modified RPE for oscillations in a narrow gap derived by Leighton in [21] as a reference solution. This equation resembles the standard RPE but with some additional terms to account for the geometrical confinement:

$$
R \ddot{R}\left[1+\frac{R}{H}\left(\ln \left[\frac{L_{c}}{H}\right]-1\right)\right]+\frac{3}{2} \dot{R}^{2}\left[1+\left(\frac{4 R}{3 H}\right)\left(\ln \left[\frac{L_{c}}{H}\right]-1\right)\right]=\frac{1}{\rho_{L}}\left[p_{B, 0}\left(\frac{R_{0}}{R}\right)^{3 \gamma_{g}}-p_{0}-\frac{2 \sigma}{R_{0}}-P(t)\right]
$$

where the overdots represent time derivatives, $H=H_{c} / 2$, and $P(t)=-p_{a c} \sin \left(2 \pi f_{a c} t\right)$. We use Eq. (10) to predict the radial motion of the acoustically driven bubble and inform our choice of forcing conditions for a given $R_{0}$. Since we are interested in inertial oscillations, we choose $f_{a c}$ to be lower than the natural frequency of the bubble $f_{0}\left(\approx 60 \mathrm{kHz}\right.$ for $\left.R_{0}=50 \mu \mathrm{m}\right)$. While Eq. (10) predicts the collapse time with reasonable accuracy, it does not provide any information on the bubble shape or jet velocities. Furthermore, it is only valid for the limiting case when the bubble is at the centre of the gap due to assumptions in its derivation [21]. Fig. 2a shows the modified RPE solution and equivalent simulation (see Section 3.1) for $H_{c}=150 \mu \mathrm{m}$ in response to the acoustic driving signal with $p_{a c}=0.95 p_{0}$ and $f_{a c}=20 \mathrm{kHz}$, shown in Fig. $2 \mathrm{~b}$.

a)



b)



Fig.2: a) Comparison of modified RPE solution and equivalent simulation for limiting case $(d=0)$ with $H_{c}=150 \mu \mathrm{m}$; b) One-cycle of the low-frequency acoustic driving signal $p_{a c}=0.95 \mathrm{p}_{0}$ and $f_{a c}=20 \mathrm{kHz}$.

\section{Results}

In this section we present results of a study of the bubble collapse under the action of the oscillatory pressure boundary for three different regimes. For each regime, we present selected instants of the pressure and velocity field evolution to demonstrate the rich array of dynamics (including necking, splitting, jetting, and collapse) observed in this confined geometry. Each frame shows a section-plane through the rotational geometry reflected about the axis of symmetry. The upper and lower borders of each frame correspond to the secondary and primary wall, respectively. The frames only show a fraction of the radial direction (where the bubble is located). Note that the colour bar ranges vary 
significantly between each frame. In some frames, the velocity scale shows extremely high maximum velocities, reaching up to $\sim 1000 \mathrm{~m} / \mathrm{s}$ (see Fig. 5). These velocities are not necessarily the maximum jet velocities, but could also be velocities of the gas inside the bubble, or bubble collapse velocities [22]. The time series in each of Figs.(4-6) starts at maximum expansion of the bubble, $R_{\max }$, the maximum spherical equivalent radius determined from the simulations. Each regime is characterised by the dimensionless gap height $\eta=H_{c} / R_{\max }$ and the normalized off-centre distance $\delta=\left(H_{c} / 2-\right.$ $h) / R_{\max }$ which is the equivalent of the stand-off distance $s=h / R_{\max }$ for collapse near a single rigid wall. The three regimes are presented according to increasing off-centre distance $\delta$, starting from the limiting case when $\delta=0$.

\subsection{Symmetric splitting with dual-jet formation}
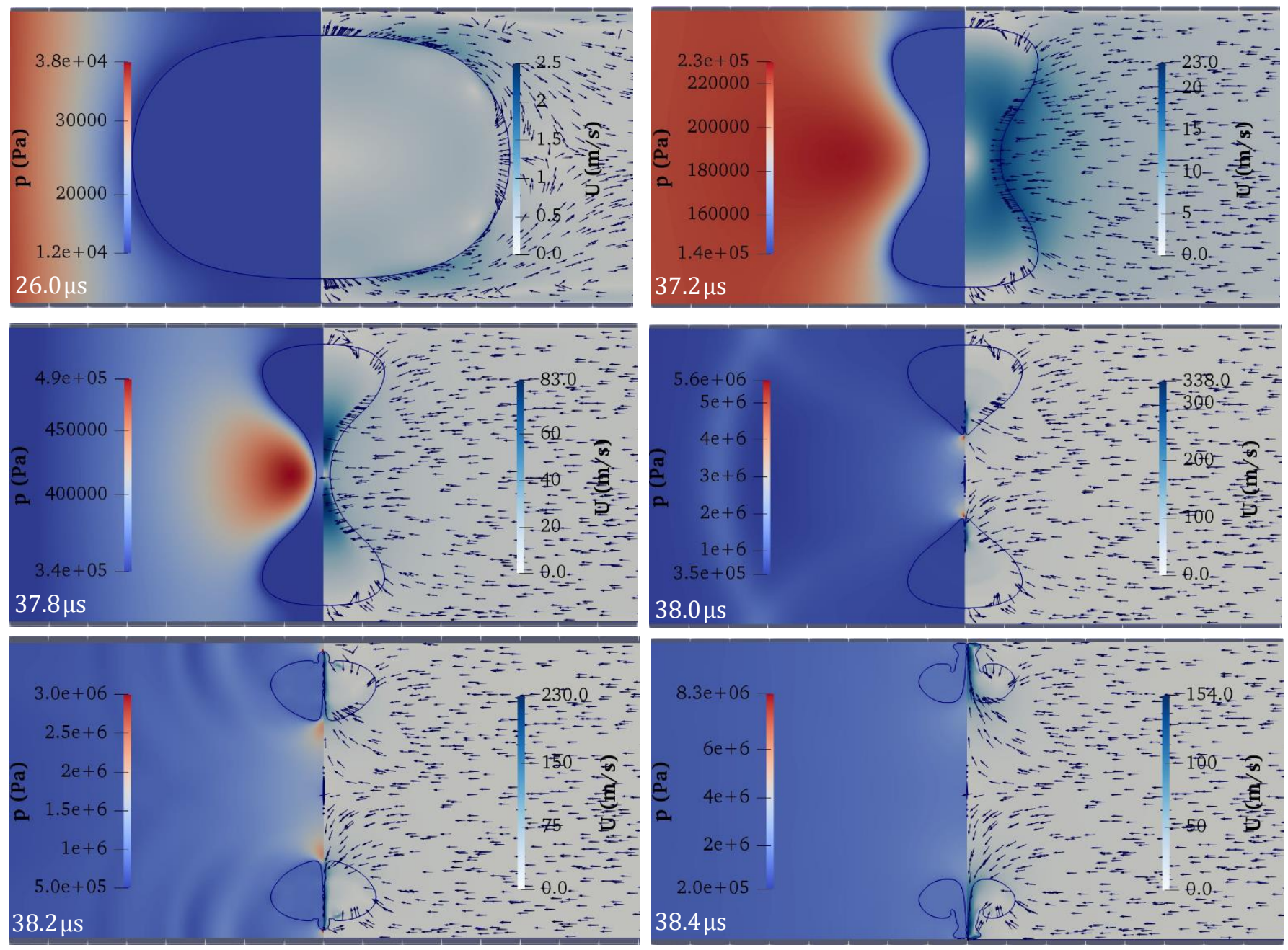

Fig. 3: Collapse sequence starting from maximum volume expansion $\left(R_{\max }\right)$ for $R_{0}=50 \mu \mathrm{m}, H_{c}=150 \mu \mathrm{m}$ and $h=75 \mu \mathrm{m}$

$(\delta=0$ and $\eta=1.83)$. Numbers in the bottom left corner of each frame indicate the time in microseconds $(\mu \mathrm{s})$.

The initially spherical bubble begins to expand under the action of the acoustic driving and reaches its maximum volume at $t=26 \mu \mathrm{s}$ with a spherical equivalent radius $R_{\max } \approx 82 \mu \mathrm{m}$, shown in Fig. 3. By this point, the sphericity of the bubble is already lost, as the radial expansion is limited by the proximity of the nearby walls which deflect the flow in the opposite direction and towards the centre of the gap. In the lateral direction, the expansion of the bubble is counteracted by the increase 
in pressure at the boundary. In fact, at $t=26 \mu \mathrm{s}$, the lateral sides have already begun to shrink as the driving pressure on the boundary increases (see Fig. 2b). When the deflected flow from the walls meets the inflow from the pressure boundary, an annular flow-focussing mechanism is created that causes the bubble to neck and form an hourglass shape at $t=37.2 \mu \mathrm{s}$. With the continued increase in the driving pressure, the necking proceeds rapidly reaching a maximum curvature at $t=37.8 \mu \mathrm{s}$. At $t=38 \mu \mathrm{s}$, the thin neck breaks, causing the bubble to split into two daughter bubbles. Since $\delta=0$ (i.e. the case has vertical symmetry), the two bubbles are equal in size. The splitting is accompanied by the emission of a spherical pressure wave (visible at $t=38 \mu \mathrm{s}$ ) as a result of the high-pressure concentration at the neck of the bubble. The annular inflow is converted into two axial jets that are also visible in the frame of $t=38 \mu \mathrm{s}$. The previously annular inflow is converted into two separate axial jets penetrating the two daughter bubbles, each directed from the centre of the gap towards the nearest rigid wall. Some small satellite bubbles can be observed at the axis of symmetry following neck closure. The axial jets penetrate the respective bubbles as is visible from the protrusions at $t=38.2 \mu$ s and proceed to impact and spread along the boundary at $t=38.4 \mu$ s.

\subsection{Asymmetric splitting with dual-jet formation}

By off-centering the initial location of the bubble $(\delta=0.29)$, the symmetry of the problem is broken, revealing a second collapse pattern in Fig.4. In this regime, a strong flow-focussing mechanism is also observed (see $t=34 \mu \mathrm{s}$ ) which causes the bubble to form a thin neck and split, as shown in frames $t=36$ and $36.2 \mu \mathrm{s}$, respectively. However, in this regime the two bubbles are of different size, the larger one being closest to the confining boundary. A highpressure region visible at $t=36.4 \mu \mathrm{s}$ is responsible for involution and jetting of the lower bubble. This larger bubble proceeds to impact the primary wall at $t=36.6 \mu \mathrm{s}$ with a very pronounced jet having a velocity of $\sim 300 \mathrm{~m} / \mathrm{s}$.
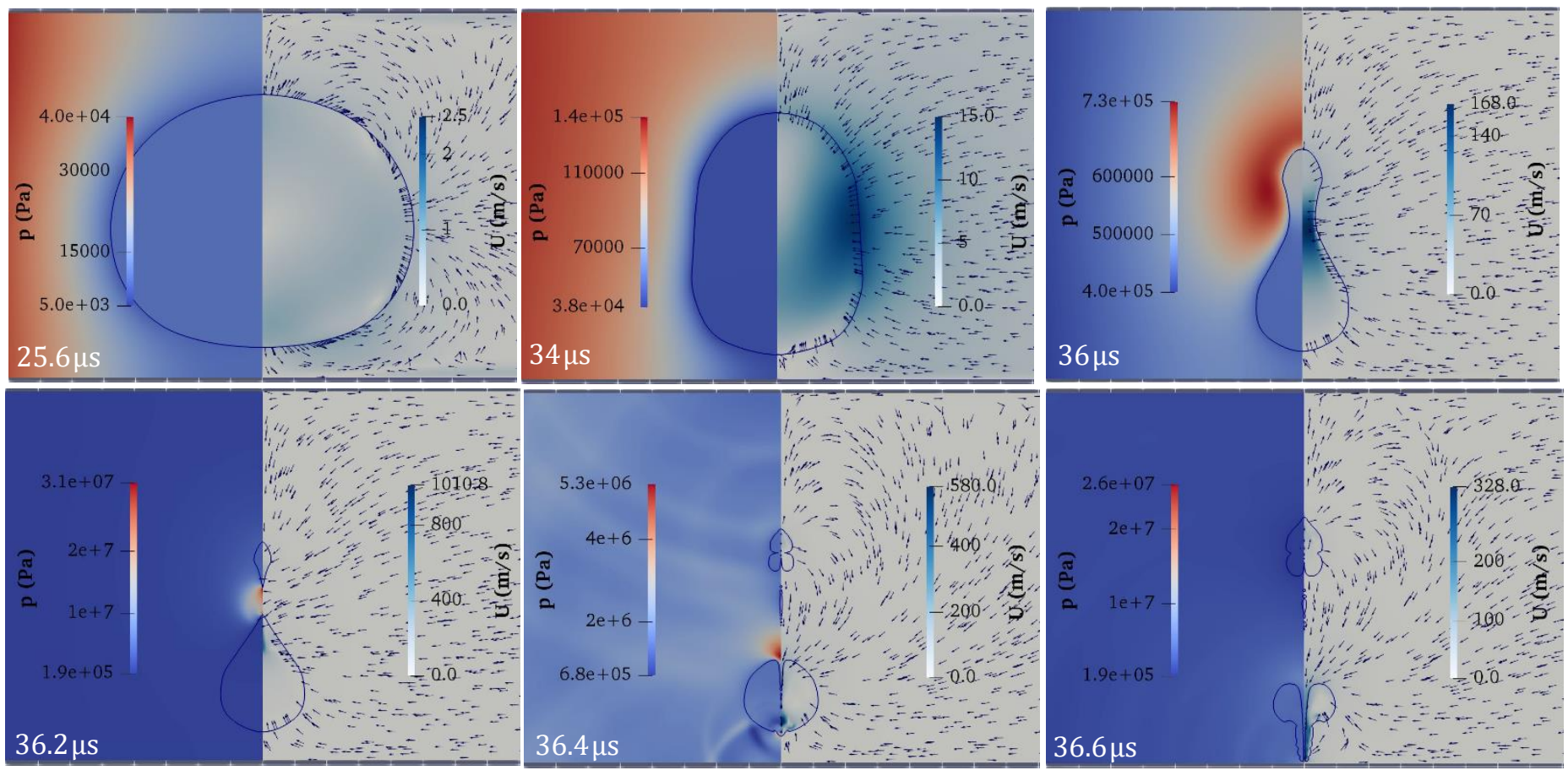

Fig. 4: Collapse sequence starting from $R_{\max }$ for $R_{0}=50 \mu \mathrm{m}, H_{c}=230 \mu \mathrm{m}$ and $h=90 \mu \mathrm{m}(\delta=0.29$ and $\eta=2.67)$.

\subsection{Collapse onto nearest wall with directed jet}

For sufficiently large $\delta$ the bubble is only minimally (if at all) affected by the secondary wall and behaves as if it were located near a single rigid wall, revealing a third collapse regime (see Fig. 5). The initially spherical bubble begins to expand under the action of the imposed boundary pressure, reaching its maximum volume with $R_{\max } \approx 87.5 \mu \mathrm{m}$ at 
$t=25 \mu \mathrm{s}$. The distal part of the bubble remains mostly spherical while the lower part is flattened out due to the presence of the nearby wall. Upon reversal of pressure driving at the boundary, the bottom interface shrinks rapidly while the distal part remains relatively spherical $(t=34 \mu \mathrm{s})$. As the bubble continues to shrink, the curvature of the distal part of the bubble increases as shown in $t=34.8 \mu \mathrm{s}$. The high-pressure region above the bubble forms an involution at $t=35 \mu \mathrm{s}$ that reveals the onset of jet formation. The jet becomes more visible at $t=35.2 \mu \mathrm{s}$ and reaches a velocity of around $288 \mathrm{~m} / \mathrm{s}$ which is considerably higher than that observed for collapse near a single rigid wall for an equivalent stand-off distance $s \approx 0.7$. The jet penetrates the bubble and impacts onto the lower wall at $t=35.6 \mu \mathrm{s}$.
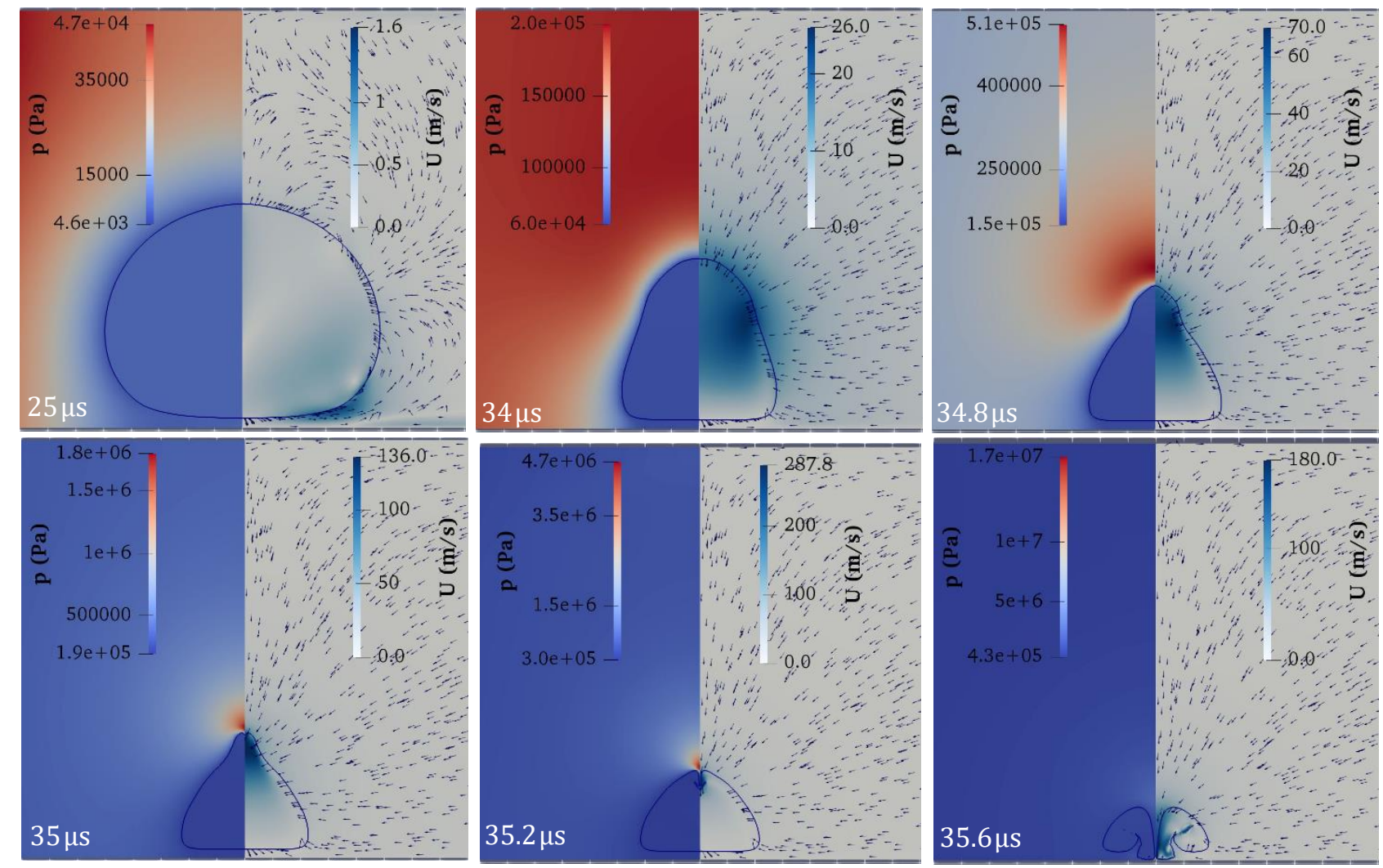

Fig. 5: Collapse sequence starting from $R_{\max }$ for $R_{0}=50 \mu \mathrm{m}, H_{c}=300 \mu \mathrm{m}$ and $h=60 \mu \mathrm{m}(\delta=1.03$ and $\eta=3.43)$.

\subsection{Influence of liquid viscosity on the dynamics}

Fig. 6 shows a representative case comparing the bubble dynamics for the same initial conditions and bubble inception position but with an increased liquid viscosity of $\mu_{L}=20 \mu_{\text {water. }}$. While the maximum volume expansion of the bubble is hardly affected (see $t \approx 25.6 \mu \mathrm{s}$ ), the overall collapse mechanism is greatly altered, becoming evident at $t=36 \mu \mathrm{s}$ when for the higher viscosity the necking is suppressed and the bubble assumes a 'teardrop' shape. For $\mu_{L}=\mu_{\text {water }}$ the bubble splits forming two opposing jets (red contours, same as Fig.4), while for $\mu_{L}=20 \mu_{\text {water }}$ the splitting is suppressed and collapse resembles that near a single rigid boundary (blue thresholds) and therefore transitions to the third regime of Section 3.3.

\section{Conclusion}

The acoustic driving introduces a further complexity in the flow-field such that the interaction of this driving with the deflected flow from the walls plays a crucial role in the collapse. The presence of confinement combined with the imposed boundary pressure result in an oscillatory non-uniform pressure distribution around the bubble causing the bubble to assume 

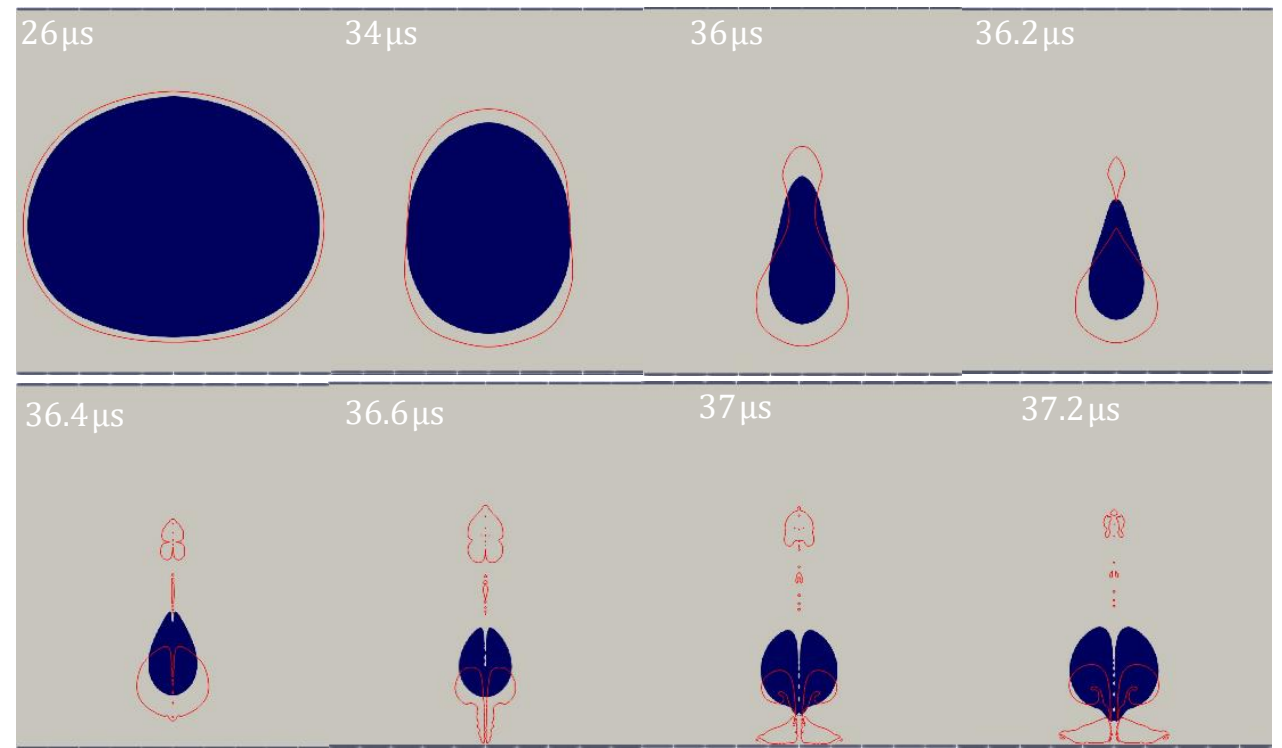

Fig. 6: Simulated bubble dynamics starting from maximum volume expansion at $R_{\max }$ for $\eta=2.64$ and $\delta=0.29$. The red contour shows the case of Fig. 4 with $\mu_{L}=\mu_{\text {water }}$ while blue threshold shows the dynamics for $\mu_{L}=20 \mu_{\text {water }}$.

a non-spherical shape at maximum expansion. The bubble collapses rapidly following one of three regimes: symmetric or asymmetric splitting with dual-jet formation, or a directed jet onto the nearest wall. The direction of the resulting jet(s) depends on the height of the gap (nondimensional $\eta$ ) and the axial position of the bubble inside the narrow gap (off-centre distance $\delta$ ). Although the presented geometry is relatively simple, we must stress that it has significant implications for realistic applications, for example in ultrasonic cleaning where the bubble activity is known to produce strong shear forces responsible for removing surface contamination [8-9,23] and in microfluidic applications for mixing and actuation [24], where the number of jets correlates with the number of boundaries confining the bubble [25]. Through careful choice of the acoustic driving and fluid properties, the desired dynamic behaviour to suit the application can be achieved.

\section{Acknowledgements}

This work is supported by Waters Corporation UK and the EPSRC under grant EP/N016602/1. The authors acknowledge the use of the Scientific Computing Research Technology Platform, and associated support services at the University of Warwick UK, in the completion of this work. The first author is indebted to Dr. Indrajit Chakraborty for interesting discussions on the subject and the authors of [9] for exchanges regarding the OpenFOAM solver.

\section{References}

[1] W. Lauterborn and T. Kurz, "Physics of bubble oscillations," Reports Prog. Phys., vol. 73, no. 10, 2010.

[2] K. Kooiman, H. J. Vos, M. Versluis, and N. De Jong, "Acoustic behavior of microbubbles and implications for drug delivery," Adv. Drug Deliv. Rev., vol. 72, pp. 28-48, 2014.

[3] W. Wiedemair, Z. Tukovic, H. Jasak, D. Poulikakos, and V. Kurtcuoglu, "On ultrasound-induced microbubble oscillation in a capillary blood vessel and its implications for the blood - brain barrier," Phys. Med. Biol., vol. 57, pp. 1019-1045, 2012.

[4] D. Rabaud, P. Thibault, M. Mathieu, and P. Marmottant, "Acoustically Bound Microfluidic Bubble Crystals," Phys. Rev. Lett., vol. 106, no. 134501, pp. 1-4, 2011.

[5] C.-D. Ohl, M. Arora, R. Dijkink, V. Janve, and D. Lohse, "Surface cleaning from laser-induced cavitation bubbles," Appl. Phys. Lett., vol. 89, p. 074102, 2006.

[6] Lord Rayleigh, "On the pressure developed in a liquid during the collapse of a spherical cavity," London, Edinburgh, Dublin Philos. Mag. J. Sci., vol. 34, no. 200, pp. 94-98, 1917. 
[7] J. R. Blake and D. C. Gibson, "Cavitation bubbles near boundaries," Annu. Rev. Fluid Mech., vol. 19, pp. 99-123, 1987.

[8] S. R. Gonzalez-Avila, E. Klaseboer, B. C. Khoo, and C.-D. Ohl, "Cavitation bubble dynamics in a liquid gap of variable height," J. Fluid Mech., vol. 682, pp. 241-260, 2011.

[9] S. R. Gonzalez-Avila, A. C. van Blokland, Q. Zeng, and C.-D. Ohl, "Jetting and shear stress enhancement from cavitation bubbles collapsing in a narrow gap," J. Fluid Mech., vol. 884, 2020.

[10] Q. Zeng, S. R. Gonzalez-Avila, and C.-D. Ohl, "Splitting and jetting of cavitation bubbles in thin gaps," J. Fluid Mech., vol. 896, p. A28, 2020.

[11] M. Koch, C. Lechner, F. Reuter, K. Köhler, R. Mettin, and W. Lauterborn, "Numerical modeling of laser generated cavitation bubbles with the finite volume and volume of fluid method, using OpenFOAM," Comput. Fluids, vol. 126, pp. 71-90, 2016.

[12] Q. Zeng, S. R. Gonzalez-Avila, R. Dijkink, P. Koukouvinis, M. Gavaises, and C.-D. Ohl, "Wall shear stress from jetting cavitation bubbles," J. Fluid Mech., vol. 846, pp. 341-355, 2018.

[13] F. Denner, F. Evrard, and B. van Wachem, "Modeling Acoustic Cavitation Using a Pressure-Based Algorithm for Polytropic Fluids," Fluids, vol. 5, no. 69, 2020.

[14] K. Schmidmayer, S. H. Bryngelson, and T. Colonius, "An assessment of multicomponent flow models and interface capturing schemes for spherical bubble dynamics," J. Comput. Phys., vol. 402, 2020.

[15] C. W. Hirt and B. D. Nichols, "Volume of Fluid (VOF) Method for the Dynamics of Free Boundaries," J. Comput. Phys., vol. 39, pp. 201-225, 1981.

[16] S. Mirjalili, S. S. Jain, and M. S. Dodd, "Interface-capturing methods for two-phase flows: An overview and recent developments," Annu. Res. Briefs, no. 1, pp. 117-135, 2017.

[17] J. U. Brackbill, D. B. Kothe, and C. Zemach, "A Continuum Method for Modeling Surface Tension," J. Comput. Phys., vol. 100, pp. 335-354, 1992.

[18] I. Chakraborty, "Numerical modeling of the dynamics of bubble oscillations subjected to fast variations in the ambient pressure with a coupled level set and volume of fluid method," Phys. Rev. E, vol. 99, no. 043107, 2019.

[19] T. Yamamoto, S. Hatanaka, and S. V. Komarov, "Fragmentation of cavitation bubble in ultrasound field under small pressure amplitude," Ultrason. - Sonochemistry, vol. 58, no. 104684, 2019.

[20] B. Han, R. Zhu, Z. Guo, L. Liu, and X. Ni, "Control of the liquid jet formation through the symmetric and asymmetric collapse of a single bubble generated between two parallel solid plates," Eur. J. Mech. / B Fluids, vol. 72, pp. 114-122, 2018.

[21] T. G. Leighton, "The inertial terms in equations of motion for bubbles in tubular vessels or between plates," J. Acoust. Soc. Am., vol. 130, pp. 3333-3338, 2011.

[22] C. Lechner, W. Lauterborn, M. Koch, and R. Mettin, "Jet formation from bubbles near a solid boundary in compressible liquid. Numerical study of distance dependence," arXiv:2005.05733v1, pp. 1-32, 2020.

[23] E. Maisonhaute, C. Prado, P. C. White, and R. G. Compton, "Surface acoustic cavitation understood via nanosecond electrochemistry . Part III : shear stress in ultrasonic cleaning," Ultrason. Sonochem., vol. 9, pp. 297-303, 2002.

[24] S.-W. Ohl and C.-D. Ohl, "Acoustic Cavitation in a Microchannel," in Handbook of Ultrasonics and Sonochemistry, Springer, Singapore, 2016, pp. 99-135.

[25] E. Zwaan, S. Le Gac, K. Tsuji, and C.-D. Ohl, "Controlled Cavitation in Microfluidic Systems," Phys. Rev. Lett., vol. 98, no. 254501, pp. 22-25, 2007. 